\title{
Article \\ CaDHN3, a Pepper (Capsicum annuum L.) Dehydrin Gene Enhances the Tolerance against Salt and Drought Stresses by Reducing ROS Accumulation
}

\author{
Yuan-Cheng Meng ${ }^{1,+}$, Hua-Feng Zhang ${ }^{1,+}$, Xiao-Xiao Pan ${ }^{1,+}$, Nan Chen ${ }^{1}$, Hui-Fang Hu ${ }^{1}$, Saeed ul Haq ${ }^{1,2}$, \\ Abid Khan 1,3 iD and Ru-Gang Chen ${ }^{1,4, *}$ \\ 1 College of Horticulture, Northwest A\&F University, Yangling 712100, China; \\ YuanchengMeng07@126.com (Y.-C.M.); 18848966687@163.com (H.-F.Z.); xiaixiao1110@163.com (X.-X.P.); \\ nanCHEN224@163.com (N.C.); huifang1919@163.com (H.-F.H.); saeed_ulhaq@nwafu.edu.cn (S.u.H.); \\ abidagriculturist@gmail.com (A.K.) \\ 2 Department of Horticulture, The University of Agriculture Peshawar, Peshawar 25130, Pakistan \\ 3 Department of Horticulture, The University of Haripur, Haripur 22620, Pakistan \\ 4 Shaanxi Engineering Research Center for Vegetables, Yangling 712100, China \\ * Correspondence: rugangchen@nwsuaf.edu.cn; Tel./Fax: +86-29-8708-2613 \\ + These authors contribute equally to this work.
}

Citation: Meng, Y.-C.; Zhang, H.-F.; Pan, X.-X.; Chen, N.; Hu, H.-F.; Haq, S.u.; Khan, A.; Chen, R.-G. CaDHN3, a Pepper (Capsicum annuum L.) Dehydrin Gene Enhances the Tolerance against Salt and Drought Stresses by Reducing ROS Accumulation. Int. J. Mol. Sci. 2021, 22, 3205. https://doi.org/10.3390/ ijms22063205

Academic Editor: Juan Manuel Ruiz Lozano

Received: 1 March 2021

Accepted: 17 March 2021

Published: 22 March 2021

Publisher's Note: MDPI stays neutral with regard to jurisdictional claims in published maps and institutional affiliations.

Copyright: (c) 2021 by the authors Licensee MDPI, Basel, Switzerland. This article is an open access article distributed under the terms and conditions of the Creative Commons Attribution (CC BY) license (https:// creativecommons.org/licenses/by/ $4.0 /)$.
Abstract: Dehydrins (DHNs) play an important role in abiotic stress tolerance in a large number of plants, but very little is known about the function of DHNs in pepper plants. Here, we isolated a $\mathrm{Y}_{1} \mathrm{SK}_{2}$-type DHN gene "CaDHN3" from pepper. To authenticate the function of CaDHN3 in salt and drought stresses, it was overexpressed in Arabidopsis and silenced in pepper through virus-induced gene silencing (VIGS). Sub-cellular localization showed that CaDHN3 was located in the nucleus and cell membrane. It was found that $\mathrm{CaDHN3-overexpressed} \mathrm{(OE)} \mathrm{in} \mathrm{Arabidopsis} \mathrm{plants} \mathrm{showed}$ salt and drought tolerance phenotypic characteristics, i.e., increased the initial rooting length and germination rate, enhanced chlorophyll content, lowered the relative electrolyte leakage (REL) and malondialdehyde (MDA) content than the wild-type (WT) plants. Moreover, a substantial increase in the activities of antioxidant enzymes; including the superoxide dismutase (SOD), peroxidase (POD), catalase (CAT), ascorbate peroxidase (APX), and lower hydrogen peroxide $\left(\mathrm{H}_{2} \mathrm{O}_{2}\right)$ contents and higher $\mathrm{O}_{2}{ }^{\bullet-}$ contents in the transgenic Arabidopsis plants. Silencing of CaDHN3 in pepper decreased the salt- and drought-stress tolerance, through a higher REL and MDA content, and there was more accumulation of reactive oxygen species (ROS) in the CaDHN3-silenced pepper plants than the control plants. Based on the yeast two-hybrid ( $\mathrm{Y} 2 \mathrm{H})$ screening and Bimolecular Fluorescence Complementation (BiFC) results, we found that CaDHN3 interacts with CaHIRD11 protein in the plasma membrane. Correspondingly, the expressions of four osmotic-related genes were significantly up-regulated in the CaDHN3-overexpressed lines. In brief, our results manifested that CaDHN3 may play an important role in regulating the relative osmotic stress responses in plants through the ROS signaling pathway. The results of this study will provide a basis for further analyses of the function of DHN genes in pepper.

Keywords: dehydrins; $C a D H N 3$; overexpression; abiotic stresses

\section{Introduction}

Plants are exposed to a large number of abiotic stresses for instance, drought and salt stresses. These stresses hinder plant growth, development, yield and severe can lead to plant death [1]. To survive in the stressed environment, plants have adapted different responses, such as the plant responses at molecular and physiological levels [2]. Many stress-inducible genes with various functions have been identified in Arabidopsis, rice, and other plants, including a number of transcription factors that regulate stress-inducible 
gene expression. The products of stress-inducible genes function both in the initial stress response and in establishing plant stress tolerance [3].

Pepper (Capsicum annuum L.) is one of the important vegetable crops in the world and is appreciated due to its unique taste and rich nutritional value [4]. During the growth and development stage, the pepper plants are easier to suffer from different stresses, including salt and drought conditions. These adverse conditions may decrease pepper seedling germination, growth, and in severe cases, accelerate pepper death. DHNs can improve plants' tolerance to abiotic stresses, prevent cell dehydration, maintain cell homeostasis, eliminate the free radicals and combine the metal ions $[5,6]$. Therefore, it is necessary to explore the stress resistance of plants and their adaptation to stressed conditions [7].

DHNs are part of group II of the late-embryogenesis-abundant (LEA) proteins. Generally, DHN proteins are regarded as a group of hydrophilic proteins which include three conserved structural domains, i.e., the $\mathrm{K}, \mathrm{Y}$, and $\mathrm{S}$ fragments. The $\mathrm{K}$-segment contains the Lys-rich structure which shapes an $\alpha$-helix in the C-terminus, that interacts with the lipid membrane or the proteins [8-10], while all the dehydrins have the K segments. However, some dehydrins contain the $S$ and $Y$ segments. According to the three conserved motifs, DHNs have been classified into five groups; namely YnSKn, YnKn, SKn, Kn, and $\mathrm{KnS}[11,12]$.

DHNs play an important role in tolerance to abiotic stresses, which can maintain cell stabilization, and protect macromolecules when subjected to drought, cold, salt and high-temperature stresses. Multiple DHN genes have been found in tomato, rice, banana, wheat, maize, and Arabidopsis, which are reported to enhance the cold and drought stresses [13-18]. DHNs play a valuable role in drought stress tolerance in a large number of plant species [8], such as HbDHN1 and HbDHN2 enhanced the plant tolerance to drought and osmotic stresses in banana [19]. Likewise, in Sorghum bicolor, the $\mathrm{Y}_{\mathrm{S}} \mathrm{K}_{2}$ Type dehydrin (SbDHN1) improved protection at high temperature and osmotic stress conditions [20], the maize KS-type dehydrin (ZmDHN13) enhanced the tolerance of transgenic tobacco to oxidative stress. However, little is known about the function of DHNs in pepper.

Thus, exploring the role of DHN genes under abiotic stresses will contribute to the regulation mechanism of DHNs. Our previous studies found 7 DHN genes in pepper [21], and previous studies divulged that DHNs are localized in cytoplasm [22], nucleus [23], mitochondria [24] and chloroplasts [25]. Although there are many reports on DHNs, however, its regulation mechanism is still unknown. In this research, we separated a pepper $\mathrm{Y}_{1} \mathrm{SK}_{2}$-type dehydrin gene $\mathrm{CaDHN} 3$ (CA02g06010 from pepper CM334 database), based on our previous study that it was strongly induced by salt and drought stresses [21]. Thus, the purpose of this study was to learn the functional role of the CaDHN3 and its regulation under salt and drought stresses. The function of the CaDHN3 was analyzed through overexpression in Arabidopsis and silencing through virus-induced gene silencing (VIGS) in pepper. The CaDHN3-transgenic Arabidopsis and CaDHN3-silenced pepper plants were subjected to salt and drought conditions. The results of this study manifested that this gene positively regulated the plants against salt and drought stresses. This study will provide useful information about the function of DHNs in pepper and other important vegetable crops.

\section{Results}

\subsection{CaDHN3 Sub-Cellular Localization}

To know the sub-cellular localization of CaDHN3 protein, the fusion protein $\mathrm{pVBG} 2307-$ CaDHN3-GFP was constructed. The GV3101 strain with pVBG2307:CaDHN3:GFP and pVBG2307:GFP (as control) vectors were rapidly expressed in the leaves of N. benthamiana plants. According to the instantaneous conversion assay, the GFP-CaDHN3 fusion protein was showed in the nucleus and the cell membrane of tobacco cells Figure 1. 


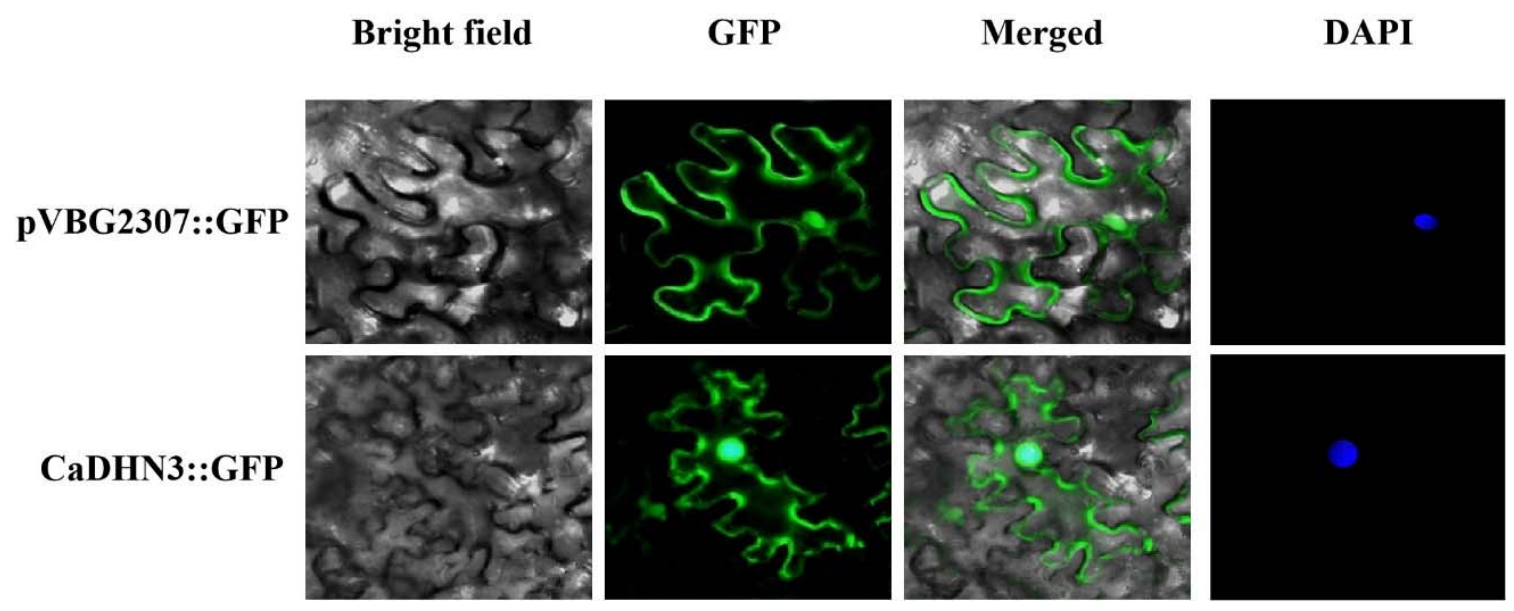

Figure 1. The sub-cellular localization of pepper CaDHN3. Agrobacterium tumefaciens strain GV3101 with pVBG2307:CaDHN3:GFP and pVBG2307:GFP (as control) vectors were transiently expressed in N. benthamiana leaves. Bars in this picture are $50 \mu \mathrm{m}$.

\subsection{Silencing of CaDHN3 Reduces Salt Stress Tolerance in Pepper}

To assess the response of CaDHN3 in salt-stress of pepper, the VIGS assay of the CaDHN3 was carried out in the pepper cold-resistant cultivar "P70". After 30 days of post-inoculation, the injected pTRV2-PDS plants were photo-bleached Figure S1A, and the relative expression of the pTRV2:00 and pTRV2:CaDHN3 were analyzed by qRT-PCR. The results indicated that the expression of $\mathrm{CaDHN} 3$ in the silenced plants decreased $86.32 \%$, which showed that $\mathrm{CaDHN} 3$ was successfully silenced in the pepper (Figure S1B). Under control conditions, no obvious differences were noticed. However, after 3 days of salt stress (300 mM NaCl), the pTRV2:CaDHN3 plant showed more wilting than the pTRV2:00 plants Figure 2A, Furthermore, to analyze the production of ROS in the CaDHN3-silenced and control (pTRV2:00) pepper plants after salt stress, the DAB and NBT staining was performed. The DAB- and NBT-stained area was substantially increased in both the pTRV2:00 and pTRV2:CaDHN3 pepper plants Figure 2B,C but the strained areas of the pTRV2:CaDHN3 plants were higher ( 26.88-50.14\%) than the pTRV2:00 Figure 2F,G. In addition, we measured the stomatal conductance of the pTRV2:CaDHN3 and pTRV2:00 plants under salt stress Figure 2D. Under normal growing conditions, there were no noticeable changes in the stomatal aperture. However, under salt stress, the pTRV2:CaDHN3 plants had an increased $(\sim 42 \%)$ average stomatal aperture than the control pepper plants Figure $2 \mathrm{H}$. Moreover, the $\mathrm{H}_{2} \mathrm{O}_{2}$ contents in the pTRV2:00 plants were evidently lowered $(\sim 22.05 \%)$ than the CaDHN3-silenced pepper plants Figure 2J; whereas, the $\mathrm{O}_{2}{ }^{\bullet-}$ content in the pTRV2:00 was significantly higher ( 24.33\%) than the CaDHN3-silenced plants (Figure $2 \mathrm{~K}$ ). These results indicated that the accumulation of ROS in the CaDHN3-silenced plants was higher than the pTRV2:00. Before salt stress, there was no evident difference in the malondialdehyde (MDA) contents and REL of the pTRV2:00 and CaDHN3-silenced plants; however, 3 days post salt stress, the CaDHN3-silenced plants showed higher MDA contents than the pTRV2:00 plants Figure 2E, and the CaDHN3-silenced plants showed higher REL contents than the pTRV2:00 plants Figure 2I. These results showed that silencing of CaDHN3 in the pepper decreased tolerance to salt stress. 


\section{A}

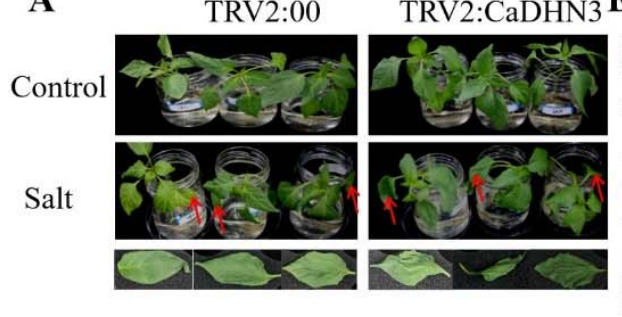

B

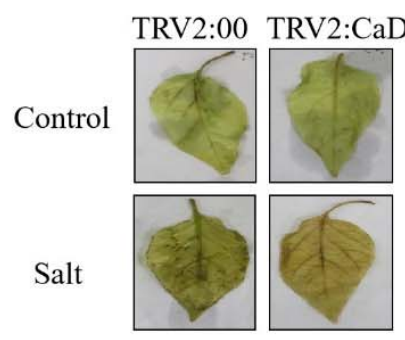

C

TRV2:00 TRV2:CaDHN3 G

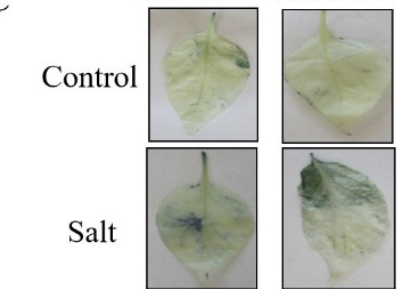

D

TRV2:00 TRV2:CaDHN3
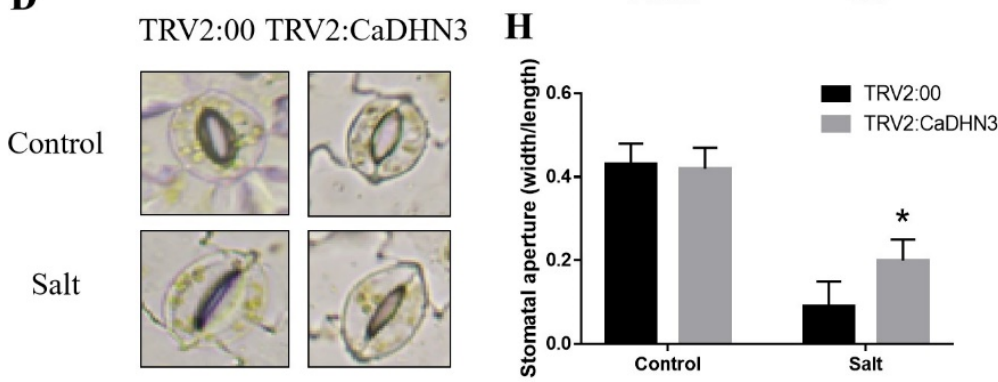
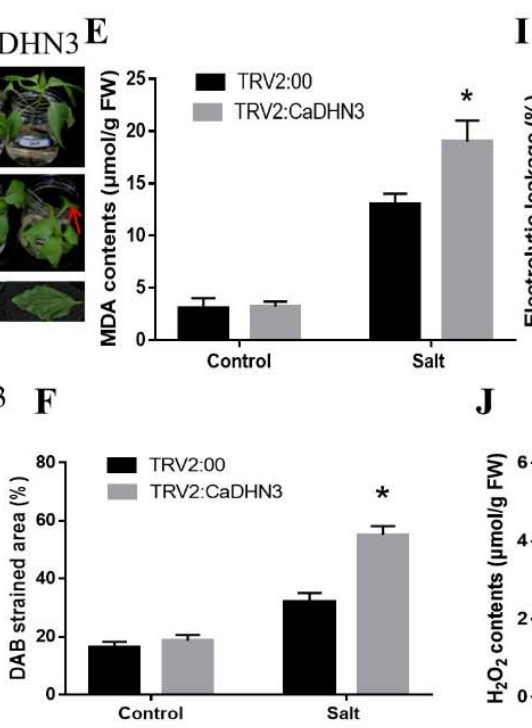

I

$\mathbf{J}$
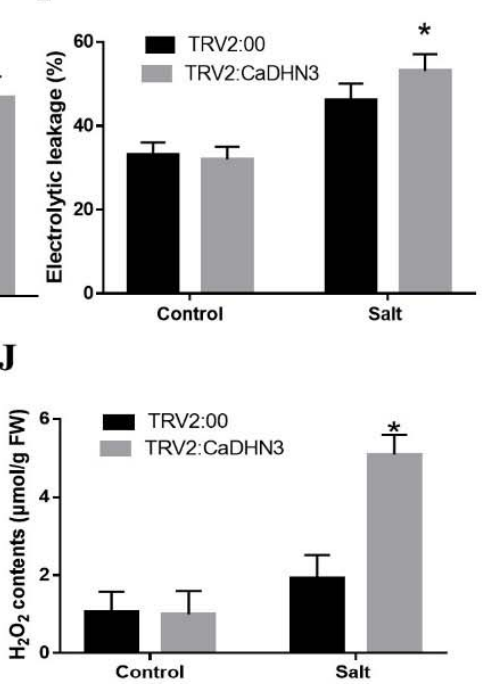

$\mathbf{K}$
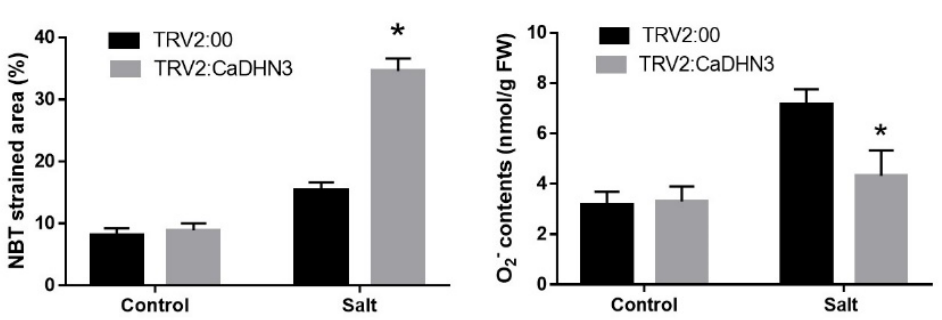

Figure 2. Physiological indies measurement under salt stress. (A) Phenotypes of silenced pepper plants under salt stress. (B) DAB staining. (C) NBT staining. (D) Stomatal conductance. (E) malondialdehyde (MDA) content. (F) \% DAB strain areas. (G) \% NBT strain areas. (H) Stomatal aperture. (I) Relative electrolyte leakage. (J) $\mathrm{H}_{2} \mathrm{O}_{2}$ contents (K) $\mathrm{O}_{2}{ }^{\bullet-}$ contents. Mean and S.D. values were obtained from three independent experiments. Asterisks indicate statistical significance $\left({ }^{*} p<0.05\right.$, Student's $t$-test) compared to control.

\subsection{Knockdown of CaDHN3 Reduced Drought-Stress Tolerance in Pepper}

To study the effect of CaDHN3 in drought-stress tolerance, the CaDHN3 was silenced through VIGS in pepper cultivar P70. Under control conditions no obvious differences were observed in the phenotypes of control and silenced pepper plants; however, 7 days postdrought stress, the pTRV2:CaDHN3 pepper plants were more wilted than the pTRV2:00 Figure 3A. Moreover, post-drought stress and the accumulation of ROS through NBT and $\mathrm{DAB}$ staining were examined in the control and CaDHN3-silenced pepper plants. The results showed that the ROS accumulation was higher in the pTRV2:CaDHN3 pepper plants than the control Figure 3B,C. The DAB and NBT strained areas of the pTRV2:CaDHN3 pepper plants were higher ( $34.99-56.20 \%)$ than the control plants Figure 3F,G. Additionally, we also measured the stomatal conductance of the CaDHN3-silenced and control pepper plants under drought stress Figure 3D. Under normal growing conditions, there were no distinct changes in the stomatal aperture. However, under drought stress, an increased 
average stomatal aperture $(\sim 52.36 \%)$ was observed in the CaDHN3-silenced pepper plants than the control Figure $3 \mathrm{H}$. The $\mathrm{H}_{2} \mathrm{O}_{2}$ contents in the $\mathrm{CaDHN} 3$-silenced pepper plants were significantly higher than in the control plants Figure $3 \mathrm{~J}$, while the $\mathrm{O}_{2}{ }^{\bullet-}$ contents of the CaDHN3-silenced plants were markedly lower than the control plants Figure 3K. These results indicated that the accumulation of ROS in the CaDHN3-silenced pepper plants was higher as compared to the control plants. Similarly, before drought stress, no significant differences in the MDA contents and REL of the control and CaDHN3-silenced pepper plants were observed. However, after drought stress, the CaDHN3-silenced pepper plants showed higher MDA and lowered REL contents than the pTRV2:00 plants Figure 3E,I). This showed that silencing of $\mathrm{CaDHN} 3$ decreased the drought stress tolerance of the pepper.

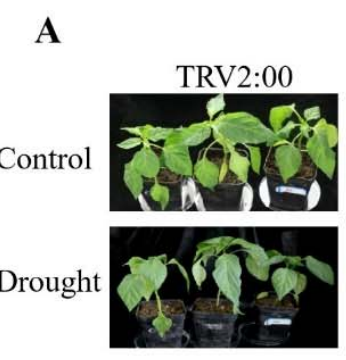

B

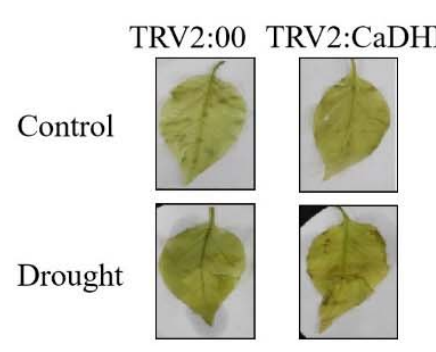

C

Control

TRV2:00 TRV2:CaDHN3

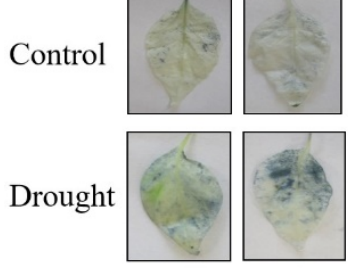

D

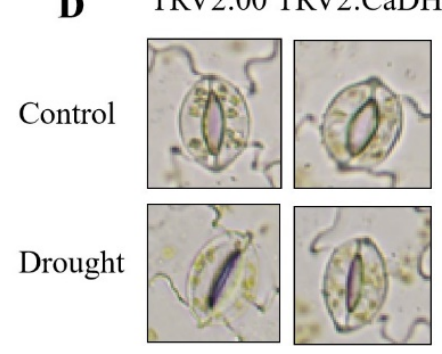

$\mathbf{E}$

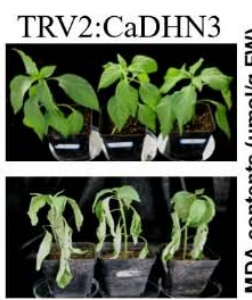

$\mathbf{F}$

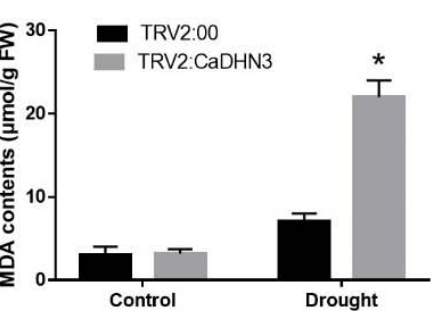

I

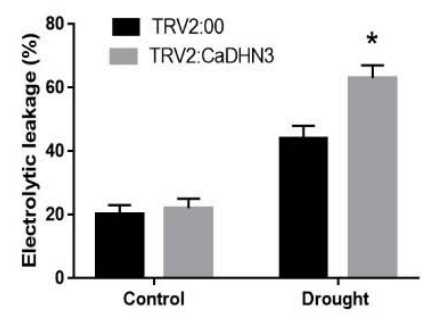

$\mathbf{J}$
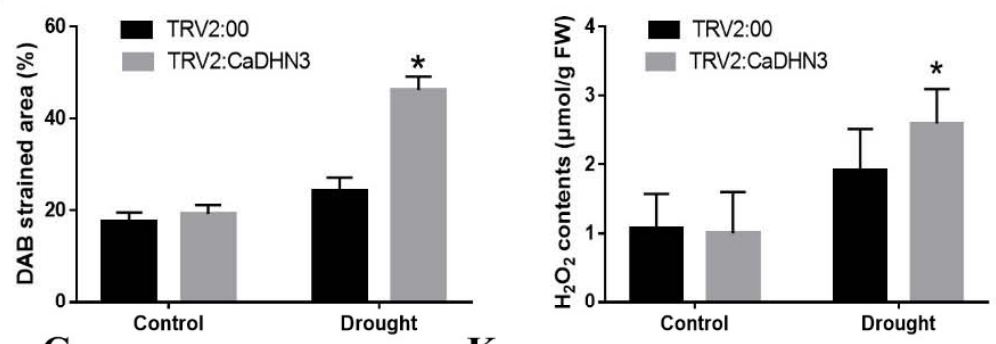

$\mathbf{K}$
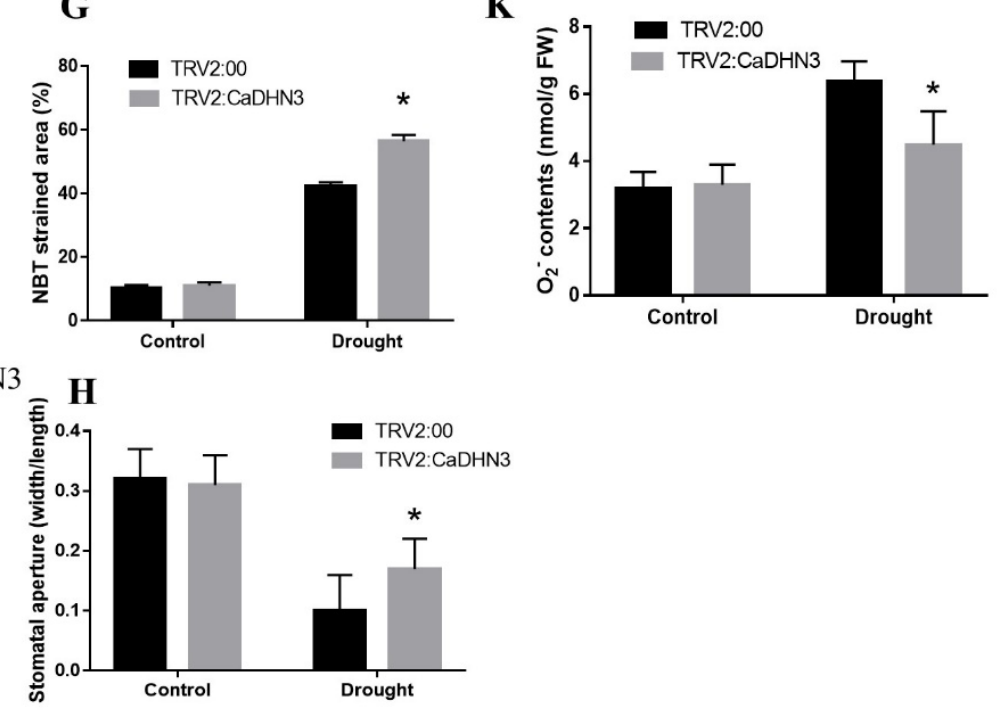

Figure 3. Physiological indices measurement under drought stress. (A) Phenotypes of silenced pepper plants under drought Scheme. (B) DAB staining. (C) NBT staining. (D) Stomatal conductance. (E) MDA content. (F) \% DAB strain areas. (G) \% NBT strain areas. (H) Stomatal aperture. (I) Relative electrolyte leakage. (J) $\mathrm{H}_{2} \mathrm{O}_{2}$ contents $(\mathbf{K}) \mathrm{O}_{2}{ }^{\bullet-}$ contents. Mean and S.D. values were obtained from three independent experiments. Asterisks indicate statistical significance $\left(^{*} p<0.05\right.$, Student's test) compared to control. 
Furthermore, the expression of CaDHN3 and ROS related genes CaAPX1(GenBank accession number: DQ002888.1), CaCAT2 (GenBank accession number: AY128694.1), CaSOD (NM_001324998.1) and CaPOD (NM_001324997.1) were strongly induced after drought and salt treatment, the expression of $\mathrm{CaDHN} 3$ in the detached leaves of control plants significantly higher than that in the detached leaves of CaDHN3-silenced pepper plants Figure 4A,F. After 3 days of salt stress and 7 days of drought stress, the expression of CaAPX1, CaCAT2, CaSOD and CaPOD were lower in the CaDHN3-silenced pepper plants than the control plants Figure 4B-E,G-J.
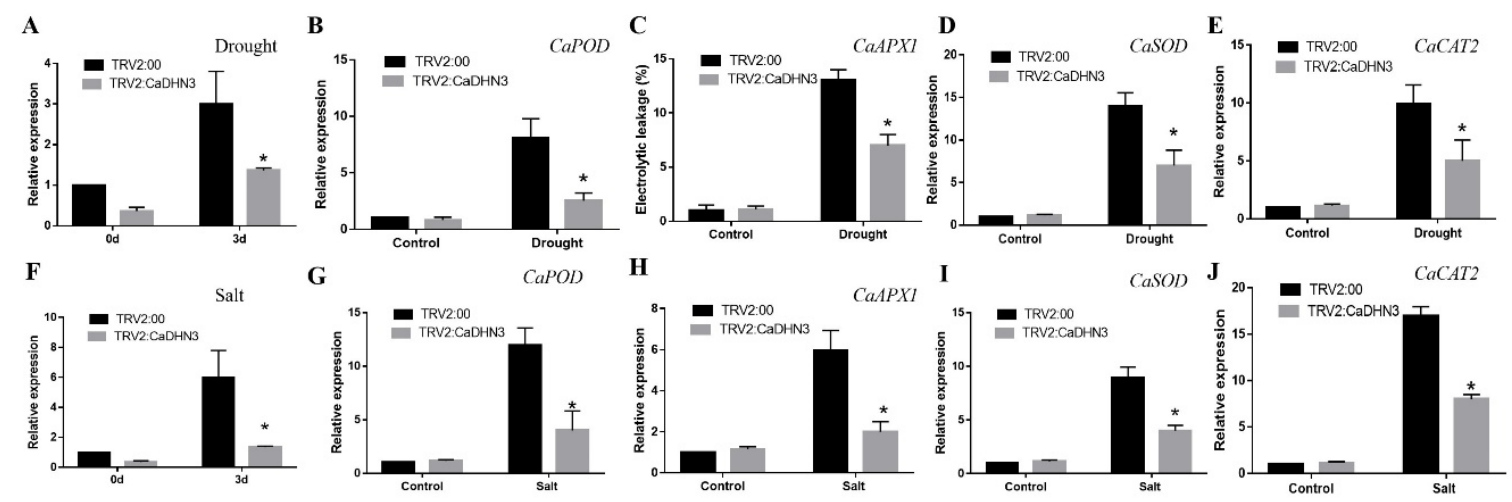

Figure 4. Reactive oxygen species (ROS) scavenging enzymes related gene expression after 3 days of salt stress and 7 days of drought stress. (A,F) relative expression of CaDHN3 under drought and salt stress. $(\mathbf{B}, \mathbf{G})$ relative expression of $C a P O D$ under drought and salt stress. $(\mathbf{C}, \mathbf{H})$ relative expression of CaAPX1 under drought and salt stress. (D,I) relative expression of $C a S O D$ under drought and salt stress. (E,J) relative expression of CaCAT2 under drought and salt stress. Mean and S.D. values were obtained from three independent experiments. Asterisks indicate statistical significance $\left({ }^{*} p<0.05\right.$, Student's test) compared to control.

\subsection{Overexpression of $\mathrm{CaDHN} 3$ in Arabidopsis Increased Salt Stress Tolerance}

To find out the role of CaDHN3 in Arabidopsis under salt stress, the CaDHN3transgenic Arabidopsis lines and WT plants were exposed to salt stress at various stages of growth. We first inspected the seed germination rates of transgenic Arabidopsis and WT plants on $\frac{1}{2}$ MS medium including different concentrations of $\mathrm{NaCl}(0,100$, and $150 \mathrm{mM})$. Similarly, the root growth of the transgenic Arabidopsis and WT plants on $\frac{1}{2}$ MS medium containing different concentrations of $\mathrm{NaCl}(0$, and $50 \mathrm{mM}$ and $100 \mathrm{mM})$ was also observed. Under normal conditions, the germination rates and root length of the transgenic Arabidopsis and the WT plants showed no significant differences, but the transgenic Arabidopsis plants showed evidently higher germination rates and root lengths than the WT plants under $\mathrm{NaCl}$ treatments Figure 5C,D. To further explore the role of CaDHN3-overexpressed Arabidopsis plants' response to salt stress, we measured the fresh weight of the transgenic and WT seedlings. It was noticed that with the increase of $\mathrm{NaCl}$ concentrations, the fresh weight of the transgenic seedlings and WT gradually decreased. However, under 50 and $100 \mathrm{mM} \mathrm{NaCl}$ concentrations, the fresh weight of the transgenic seedlings was significantly higher ( $\sim 40.2-42.46 \%$ and $\sim 20.26-21.37 \%$ ) than the WT seedlings Figure 5E. These results demonstrated that ectopic expression of $\mathrm{CaDHN} 3$ enhanced the salt stress tolerance in Arabidopsis. 
A

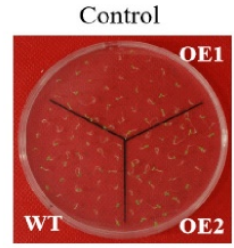

Control

B

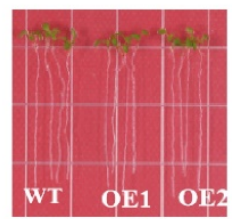

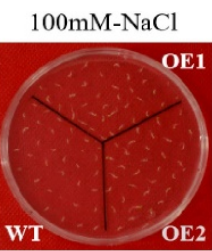

$50 \mathrm{mM}-\mathrm{NaCl}$

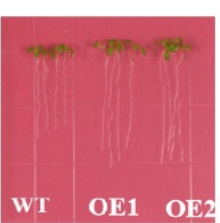

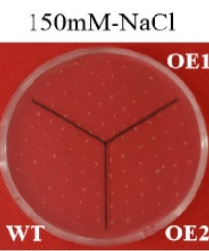

$100 \mathrm{mM}-\mathrm{NaCl}$

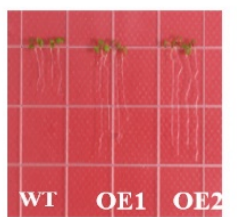

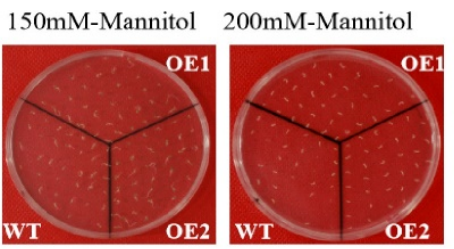

100mM-Mannitol 150mM-Mannitol

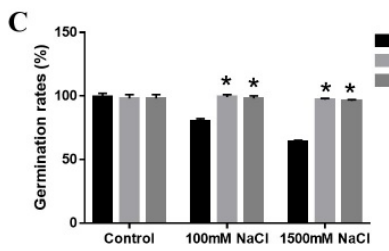

D

${ }_{T \rightarrow 1}^{5}$
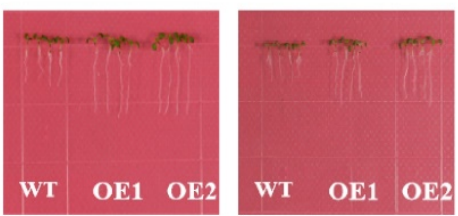

WT OE1 OE2

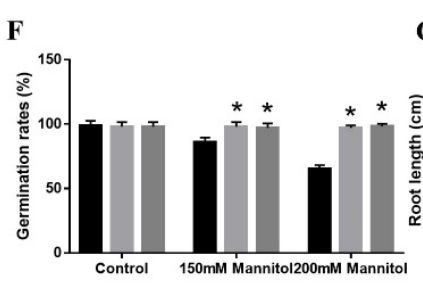

G
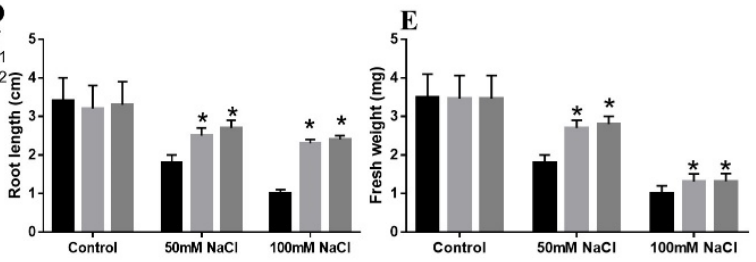

$\mathbf{H}$

${ }^{5}$
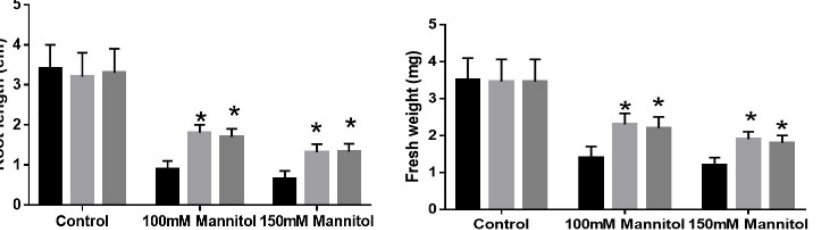

Figure 5. Analyses of salt and osmotic resistance of CaDHN3-overexpressed transgenic Arabidopsis and WT Arabidopsis plants. (A) Phenotypes of seed germination rates. (B) Root lengths. (C,F) Germination rates. (E,H) Fresh weights. (D,G) Root lengths. Mean and S.D. values were obtained from three independent experiments. Asterisks indicate statistical significance $\left({ }^{*} p<0.05\right.$, Student's test) compared to control.

\subsection{Overexpression of CaDHN3 in Arabidopsis Increased Drought Tolerance}

To authenticate the mechanism of CaDHN3-overexpressing Arabidopsis plants to drought stress, the transgenic and WT plants were subjected to drought conditions at different germination and mature stages. The germination rates of the transgenic Arabidopsis and WT plants were measured on 1/2 MS solid medium with different concentrations of mannitol (0,150, and $200 \mathrm{mM})$. The transgenic Arabidopsis plants showed markedly higher germination rates and root lengths than the WT plants under mannitol treatments Figure 5A,B. While under normal conditions, the germination rates and root length of the transgenic Arabidopsis and WT plants showed no observable differences, but under mannitol treatments, the transgenic plants revealed higher germination rates and root lengths than the WT plants Figure 5F,G. To know the role of the CaDHN3-overexpressed Arabidopsis plants in response to drought stress, the fresh weight of transgenic Arabidopsis seedlings and WT plants were measured. It was found that with the increase in mannitol concentrations, the fresh weights of the transgenic and WT plants were decreased, but the transgenic plants revealed higher fresh weights than WT plants Figure 5H. These results indicated that overexpression of $\mathrm{CaDHN} 3$ enhanced the drought stress tolerance in Arabidopsis.

Then we also determined the salt and drought stress tolerance of the transgenic and WT lines under soil-grown conditions. We found that the growth of transgenic and WT plants has no difference under normal growing conditions Figure 6A. However, after $300 \mathrm{mM} \mathrm{NaCl}$ treatment for 7 days, the leaves of the transgenic plants were gradually yellowed, but the leaves of the WT plants became shrank and even death occurred. After 
$300 \mathrm{mM} \mathrm{NaCl}$ treatment for 15 days, the WT plants died, but the transgenic plants survived and were pale green Figure 6B. After drought stress for 15 days, the WT plants showed serious wilting as compared to the transgenic plants. While, after re-watering for 3 days, the transgenic lines showed a higher survival and recovery rate than the WT plants Figure $6 \mathrm{C}$. We also found that the chlorophyll content of the transgenic plants was significantly higher under salt ( 40.46-42.39\%) and drought ( 20.26-24.46\%) stresses than the WT plants Figure $6 \mathrm{H}$.

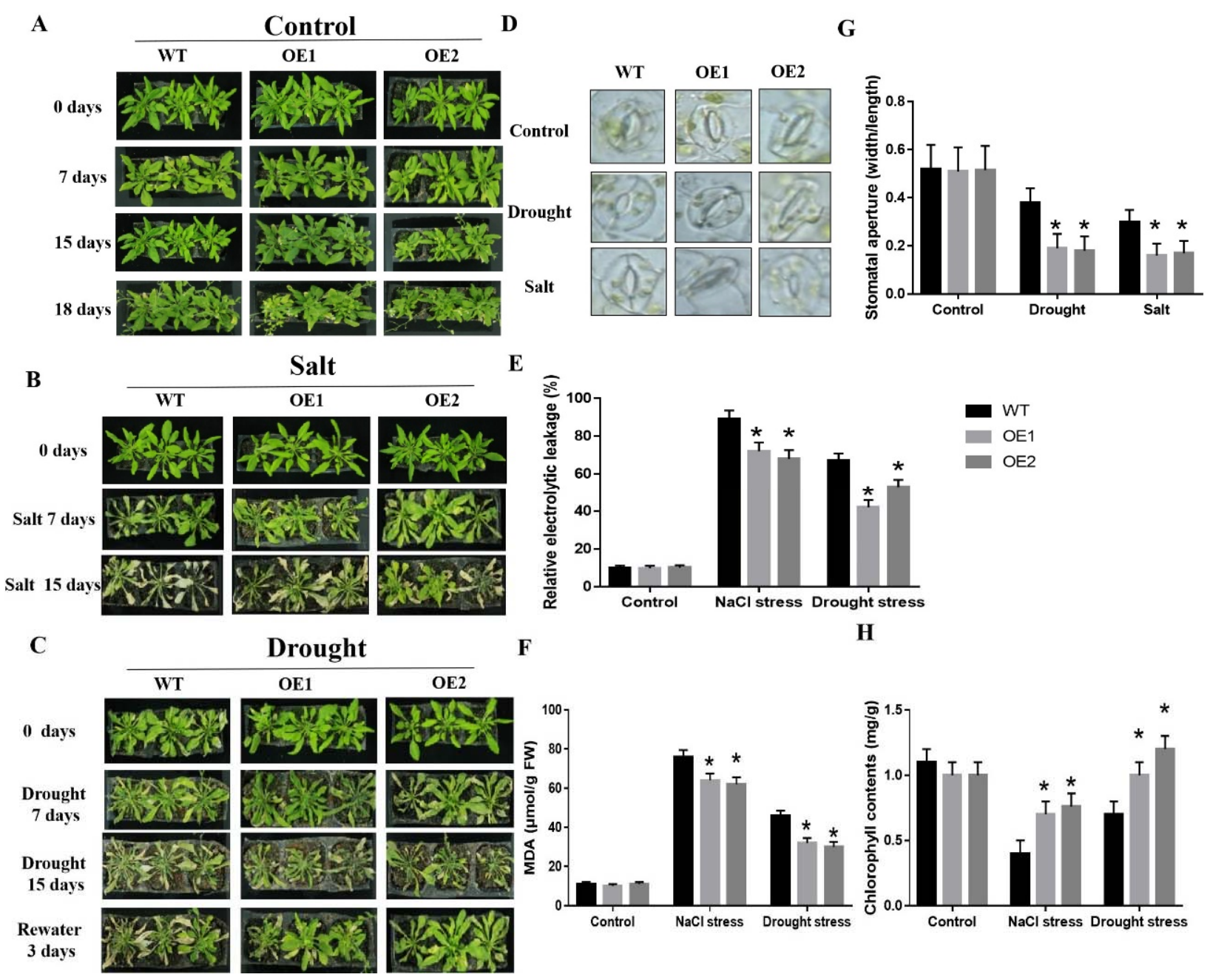

Figure 6. Overexpression of CaDHN3 in Arabidopsis enhanced the resistance of Arabidopsis to salt and drought stresses. (A) Phenotypes under normal conditions. (B) Phenotypes under salt stress. (C) Phenotypes of under drought stress. (D) and (G) Stomatal aperture of CaDHN3 transgenic and WT plants in response to salt and drought stress. (E) Electrolytic leakage rate. (F) MDA content. (H) Chlorophyll content. Mean and S.D. values were obtained from three independent experiments. Asterisks indicate statistical significance $\left({ }^{*} p<0.05\right.$, Student's test) compared to control.

To further confirm the roles of CaDHN3 in drought and salt stress tolerance, MDA contents and REL were determined under normal and osmotic stress conditions. The MDA contents and REL of the WT and transgenic plants showed no difference under normal growing conditions, but under salt stress conditions the transgenic Arabidopsis plants showed lower MDA contents and REL than the WT plants. Similarly, under drought stress the transgenic Arabidopsis plants showed lower MDA contents and REL than the WT plants Figure 6E,F. It showed that ectopic expression of that CaDHN3 increased the salt and drought stresses tolerance in Arabidopsis. 
Besides, we also measured the stomatal conductance of the transgenic and WT Arabidopsis plants under salt and drought stresses Figure 6D. Under normal conditions, the WT and transgenic plants showed no distinct difference in the stomatal aperture. However, under salt and drought stress conditions, the CaDHN3-overexpressed plants had a reduced $(\sim 40.0-60.44 \%)$ average stomatal aperture than the WT plants Figure 6G. These results showed that $\mathrm{CaDHN} 3$ promoted drought- and salt-induced stomatal closure.

\subsection{CaDHN3 Overexpression Relieves ROS Accumulation}

To know if the increased drought and salt tolerance is consisted with the ROS accumulation, we performed DAB and NBT staining also determined the $\mathrm{H}_{2} \mathrm{O}_{2}$ and contents, respectively. Under normal growing conditions, there were no distinct changes in the ROS accumulation of the transgenic and WT plants. However, under salt and drought stresses, the transgenic plants gathered little $\mathrm{H}_{2} \mathrm{O}_{2}$ and higher $\mathrm{O}_{2}{ }^{\bullet-}$ contents than the WT, as little blue or brown colors of DAB or NBT staining were detected in the leaves Figure 7A,B.

A

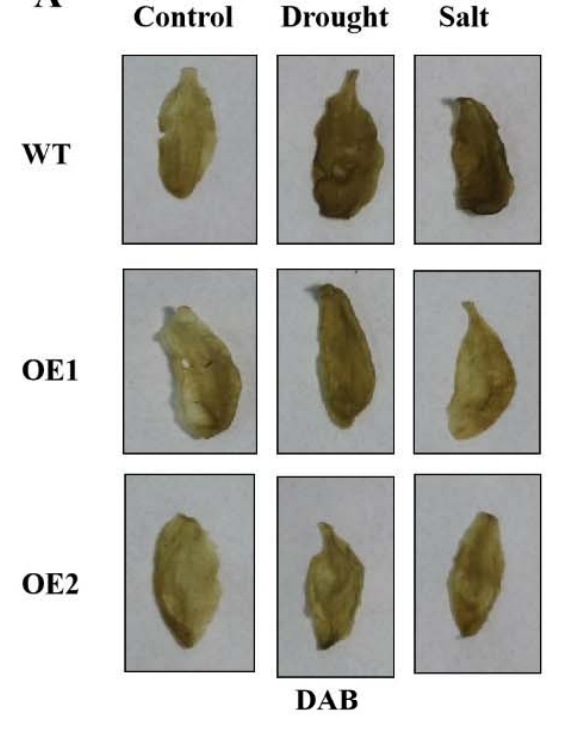

C

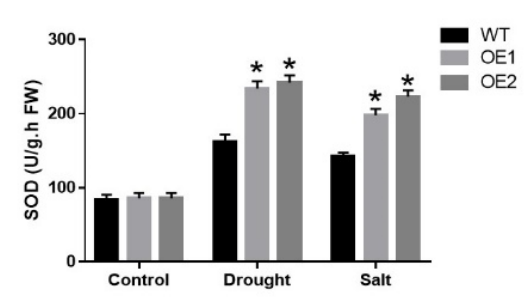

$\mathbf{F}$

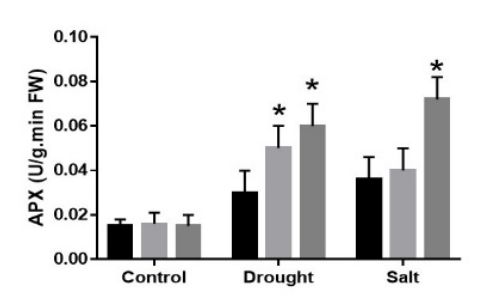

G
B
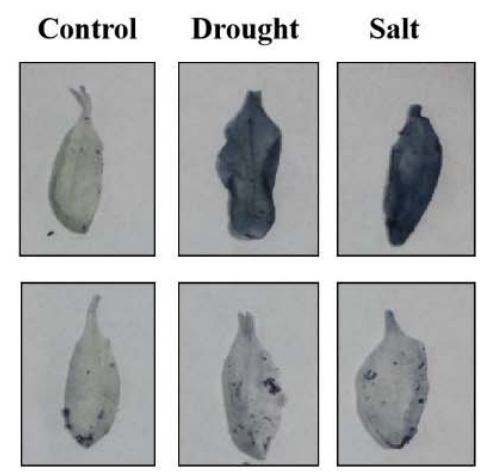

OE2
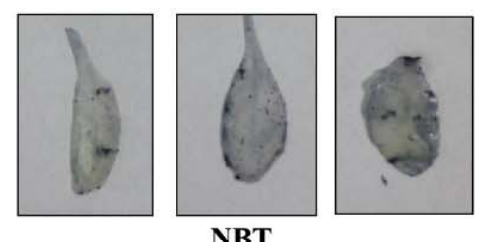

$\mathbf{E}$

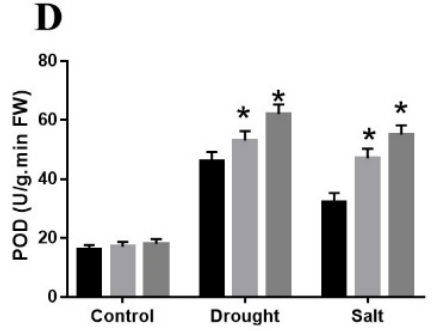

$\mathbf{H}$
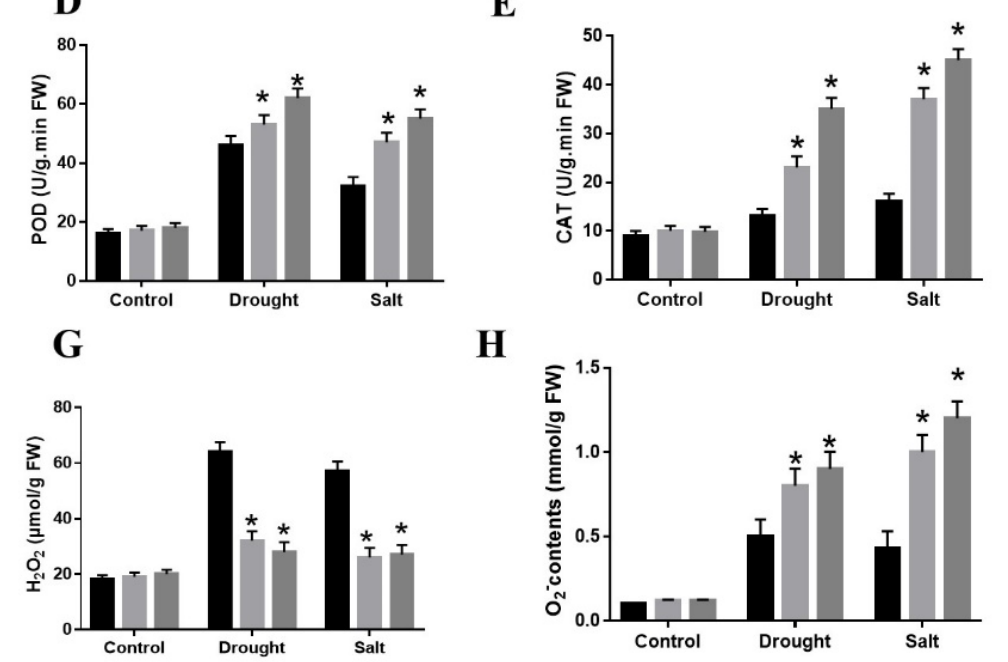

Figure 7. Overexpression of CaDHN3 enhanced ROS scavenging ability in transgenic Arabidopsis. (A,B) DAB and NBT staining. (C) Superoxide dismutase (SOD) activity. (D) Peroxidase (POD) activity. (E) Catalase (CAT) activity. (F) Ascorbate peroxidase (APX) activity. (G) $\mathrm{H}_{2} \mathrm{O}_{2}$ contents. (H) $\mathrm{O}_{2}{ }^{\bullet-}$ contents. Mean and S.D. values were obtained from three independent experiments. Asterisks indicate statistical significance $\left({ }^{*} p<0.05\right.$, Student's test) compared to control. 
It is known that superoxide dismutase (SOD), peroxidase (POD), catalase (CAT), and ascorbate peroxidase (APX) play a vital role in ROS homeostasis during $\mathrm{NaCl}$ and drought stresses by scavenging ROS. Next, we measured SOD, POD, CAT and APX enzymatic activity under drought or salt stresses, the SOD, POD, CAT and APX showed significantly higher activity in the transgenic lines compared to WT plants under salt and drought treatments Figure 7C-F. Then we determined in situ accumulation of two major ROS, $\mathrm{H}_{2} \mathrm{O}_{2}$ and $\mathrm{O}_{2}{ }^{\bullet-}$ Quantitative results also showed that cellular $\mathrm{H}_{2} \mathrm{O}_{2}$ contents in the overexpressing lines were lower than in the WT, however, $\mathrm{O}_{2}{ }^{\bullet-}$ contents was higher in the overexpressing lines than WT Figure 7G,H. These results showed that CaDHN3 overexpression can relieve ROS accumulation of the transgenic plants through increasing the activities of these anti-oxidative enzymes.

\subsection{CaDHN3 Overexpression Activated the Expression of Relative Stress-Responsive Genes}

To further understand the regulating mechanisms of $C a D H N 3$ in drought and salt stresses, the expression of stress-responsive genes including COR47, DREB2A, ERD11 and $R D 29 B$ were measured in CaDHN3-overexpressed and WT plants by QPCR Figure 8. In addition, the expression level of drought and salt stress-related genes COR47, DREB2A, $E R D 11$ and $R D 29 B$ were strongly induced in transgenic Arabidopsis after drought and salt treatment. The expression level of the COR47, DREB2A, ERD11 and RD29B genes were higher in transgenic lines than that in WT. These results suggest that $\mathrm{CaDHN} 3$ positively activates drought and salt-responsive gene expression in transgenic Arabidopsis.

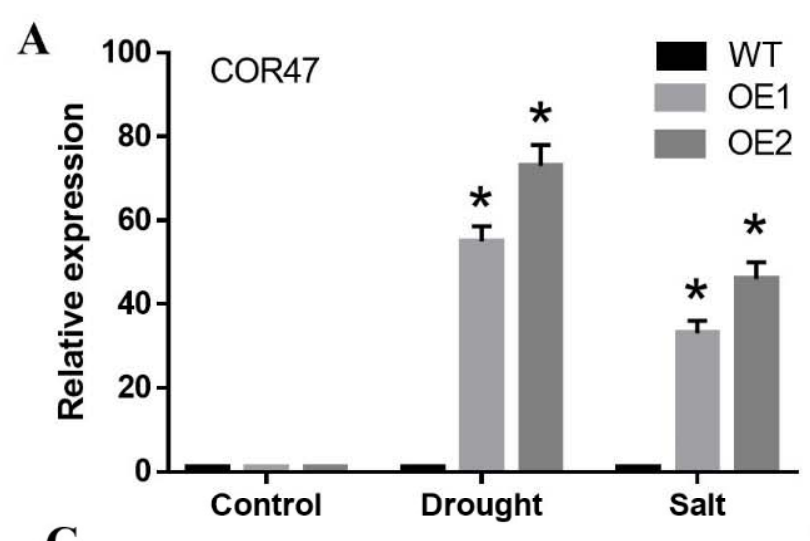

B
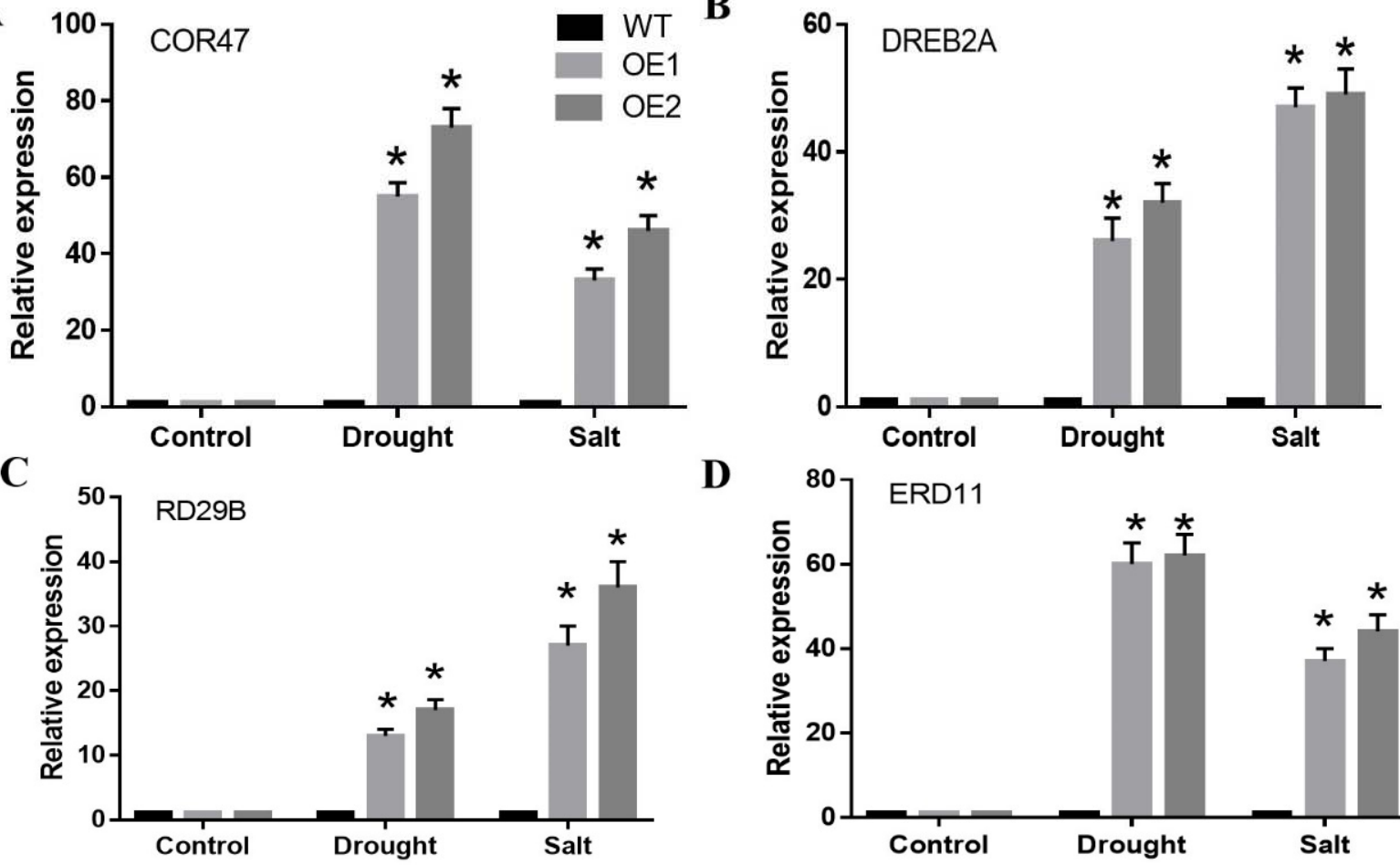

D

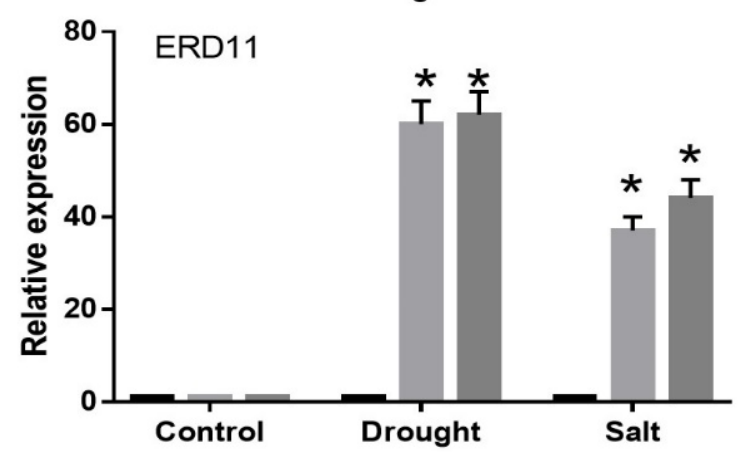

Figure 8. Overexpression of $\mathrm{CaDHN} 3$ activated the relative expression of the stress responsive genes. (A) Relative expression of COR47 under drought and salt stress. (B) Relative expression of DREB2A under drought and salt stress. (C) Relative expression of RD29B under drought and salt stress. (D) Relative expression of ERD11 under drought and salt stress. Mean and S.D. values were obtained from three independent experiments. Asterisks indicate statistical significance $\left({ }^{*} p<0.05\right.$, Student's test) compared to control.

\subsection{CaDHN3 Interact with CaHIRD11 Protein}

The ORF fragments of the proteins CaHIRD11 was inserted into pGADT7 vectors, and transferred to yeast strainsY2H Gold also containing pGBK7-CaDHN3. As shown 
in Figure 9, the co-transformed CaDHN3-BD and CaHIRD11-AD were grown on SDLeu-Trp, SD/-Trp/-Leu/-His/-Ade. The results showed that CaDHN3 interacted with CaHIRD11 protein. To further confirm CaDHN3 interacts with CaHIRD11 protein, we also performed BiFC experiment in tobacco leaves. The results were consistent with $\mathrm{Y} 2 \mathrm{H}$, CaDHN3 interacted with CaHIRD11 protein in the plasma membrane Figure 9.

A pGBKT7-P53+pGADT7-T
pGBKT7-lam+pGADT7-T
pGBKT7-CaDHN3+
pGADT7-CaHIRD11

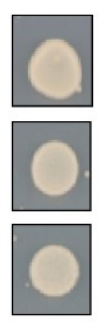

$\mathrm{SD} /-\mathrm{Trp} /-$ Leu
B

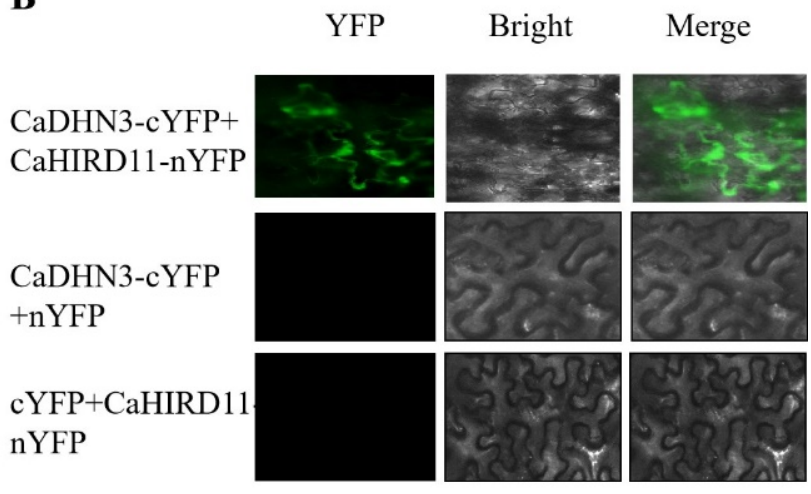

Figure 9. The interactions of CaDHN3 with CaHIRD11. (A) Interactions of CaDHN3 with CaHIRD11 by yeast two-hybrid assay, pGBKT7-53 + pGADT7-T as positive control, the pGBKT7-Lam + pGADT7-T as negative control. (B) BiFC assays were used to measure the interaction between CaDHN3 with CaHIRD11. Bars in this picture are $50 \mu \mathrm{m}$.

\section{Discussion}

A number of DHN genes have been found and their regulatory mechanisms have also been studied and analyzed [26-29]. The protein structure of DHNs is divided into three conserved motifs, K, Y, and S fragments. The existence or deficiency of the YSK fragments is connected with the localization of the DHN in the cell [6]. YnKn, YSK, and Kn DHNs have been found that localizes to the cytoplasm and cell nucleus [30,31]. SKn DHNs are found to localize in the plasma membrane [32,33] However, KnS DHN was found in the mitochondrial [33]. No DHN Y-segment was found to localize in the membrane, which speculates that the Y-segment does not play a valuable role in cell membrane protection [6]. $\mathrm{CaDHN} 3$ contained one $\mathrm{S}$ fragment at the $\mathrm{N}$-terminal and two $\mathrm{K}$ fragments at the $\mathrm{C}$-terminal, thus, were named $\mathrm{Y}_{1} \mathrm{SK}_{2}$-type DHN. To explore the function and the regulatory pathway of different stresses, we amplified a $\mathrm{Y}_{1} \mathrm{SK}_{2} \mathrm{DHN}$ gene (CaDHN3) from pepper, based on our previous study [21]. Many DHNs have been found that can be induced under salt and drought stresses. Overexpression of DHNs genes in different plant species, such as Arabidopsis, wheat and tomato enhanced the drought, salt, and cold stresses tolerance. In different stages of plant growth and development, the WT and transgenic Arabidopsis showed no differences under normal growing conditions. However, when subjected to salt and drought stresses, the transgenic Arabidopsis lines were more sensitive to both the morphological and physiological indicators than the WT. In a previous study, silencing of $\mathrm{CaDHNs}$ through VIGS, decreased cold and salt stress tolerance, and our results also showed that silencing of CaDHN3 decreased the drought and salt stress tolerance [21], In the current study, we carried out further research of the CaDHNs for the drought and salt assay, and will offer new insights and discoveries.

To explore the functional role of $\mathrm{CaDHN} 3$, this gene was overexpressed in Arabidopsis. Overexpression of the CaDHN3 enhanced the drought and salt tolerance of Arabidopsis Figure 5, while silencing of CaDHN3 in pepper decreased the drought and salt stress tolerance. The transgenic Arabidopsis lines also had higher chlorophyll contents, lower electrolyte leakage, higher germination rate and root lengths than the WT Arabidopsis plants under salt, and drought stresses Figures 4 and 5. Likewise, CaDHN3-silenced pepper 
plants showed higher $\mathrm{H}_{2} \mathrm{O}_{2}$ contents lower $\mathrm{O}_{2}{ }^{\bullet-}$ contents than the control plants. These findings demonstrated that $\mathrm{CaDHN} 3$ acted as a positive regulator.

The mechanisms of improving drought and salt tolerance in Arabidopsis might be beneficial and brings changes in the morphological, physiological and biochemical aspects. In this research, the first change was the transgenic seedlings showed longer roots than the WT Figure 4, indicating that $\mathrm{CaDHN} 3$ may regulate root systems under salt and osmotic stresses, consequently improving stress tolerance. Another change could be the physiological and biochemical characteristics. When plants are subjected to adverse conditions the chlorophyll contents tend to decrease [34]. While in this study, the chlorophyll content of CaDHN3 transgenic plants was obviously higher than the WT after salt or drought stress treatments Figure 5H. MDA content and REL are the important physiological indicators of plant resistance [35]. Therefore, we determined MDA content and REL to explore the role of $\mathrm{CaDHN} 3$ overexpression in reducing the membrane damage under drought and salt conditions. The REL of WT plants was higher than the transgenic plants under salt and drought stresses Figure 5E, and the MDA content in WT plants was obviously higher than the transgenic plants under salt and drought stresses Figure 5F. In general, these results indicated that $\mathrm{CaDHN} 3$-overexpressed plants may decrease the lipid peroxidation as compared to the WT plants under salt or drought conditions. Thus, we speculate that the ectopic expression of CaDHN3 in Arabidopsis enhanced the tolerance to salt and drought stresses through the increase of root length and maintained cell membrane stabilization.

ROS scavenging plays a vital in maintaining ROS with a low level in stressful conditions and prevents oxidative harm. Antioxidant enzymes take part in ROS detoxification and thus play a crucial part in confronting abiotic stresses. In this research, DAB, NBT staining and quantitative results showed that overexpression of $\mathrm{CaDHN} 3$ caused the reduction of ROS, whereas silencing of CaDHN3 led to the accumulation of more ROS under salt and drought stresses Figures 2, 3 and 7. According to the observed results, increasing antioxidant capacity and reducing ROS accumulation may be important mechanisms that improved salt and drought tolerance of $\mathrm{CaDHN} 3$-overexpressing transgenic plants.

Plants have the enzymatic antioxidant prevent systems to protect cells from oxidative damage by scavenging ROS, such as POD, SOD, CAT and APX. CaDHN3-overexpressing plants had higher activities of POD, SOD, CAT and APX after salt and drought stress, suggesting that they have more enzymatic antioxidant prevent systems compared to WT plants Figure 7. These results suggest that the overexpression of CaDHN3 impeded ROS damage under salt and drought stress by decreasing MDA and REL levels and enhancing POD, SOD, CAT and APX activities. In this study, the expression level of the COR47, DREB2A, ERD11 and RD29B genes were strongly induced in the CaDHN3 transgenic Arabidopsis than in WT under salt and drought stress Figure 8. Therefore, these results suggested that $\mathrm{CaDHN} 3$ may be involved in plant salt and drought stress tolerance by modulating the expression level of stress-related genes. Along with these stress-related genes, we also measured the expression of CaCAT2, CaSOD, CaAPX1 and $\mathrm{CaPOD}$, which were related to ROS-scavenging enzymes after salt and drought stresses. In addition, these genes in the CaDHN3-silenced plants were lower than pTRV2:00 plants under salt and drought stresses Figure 3. The results of this research revealed that CaDHN3 gene maintained the cell membrane stability, averted lipid peroxidation and reduced the accumulation of ROS, thereby playing a valuable role in salt and drought stress tolerance. Therefore, it is speculated that $\mathrm{CaDHN} 3$ regulates the plant tolerance to salt and drought stresses possibly through ROS scavenging, and this needs further experimental evidence.

As reported by Xie et al. [36] that DHN MtCAS31 interacted with the ICE1 transcription factor and enhanced drought tolerance, which showed that DHNs acted as chaperons under abiotic stresses to protect plant better growth. Chaperons can impede useless proteins aggregation and form complexes with target proteins. It is difficult for DHNs to form an interaction with other proteins, explaining why some DHNs functions are based on the protein-protein interactions. 
$\mathrm{Y} 2 \mathrm{H}$ assays indicated the interaction between CaDHN3 and CaHIRD11 protein Figure 9A. Furthermore, BiFC showed that their interaction was located on the plasma membrane Figure 9B. These results suggested that CaDHN3 enhances the tolerance against salt and drought stresses by interacting with CaHIRD11. HIRD belongs to the SK-type DHN, DHN has been proposed to play fundamental roles in the protection of cellular components. Although various stresses cause the gene expression of DHNs, the cold is a major cue for DHN accumulation in plants [6], and transgenic plants expressing DHN genes were more cold tolerant than the corresponding wild-type plants suggesting that DHNs might prevent cellular damage due to cold [37]. In Arabidopsis we found AtHIRD11 showed cryoprotective activities for lactate dehydrogenase in a hydrophobic residue-dependent manner [38], DHNs are intrinsically disordered proteins with low contents of hydrophobic amino acids, the hydrophobic residues can play important roles in the cryoprotective activities of DHNs.

At present, we know a little about the signaling pathways of pepper dehydrins. In the previous study, we found CaDHN3 can be induced by both ABA and SA treatments [21]. So, we suggested overexpression $\mathrm{CaDHN} 3$ can enhance salt and drought stresses tolerance through abscisic acid (ABA) and salicylic acid (SA) signaling pathways. In the future study, we will verify it by measuring the germination rate under ABA and SA treatments. Taken together, these findings indicated that $\mathrm{CaDHN} 3$ may act as a positive regulator against salt and drought stresses by reducing ROS accumulation. This research will provide a base for further study to understand the role of the DHN genes in solanaceous and other crops for adaptability to different stresses.

\section{Materials and Methods}

\subsection{Plant Materials and Growth Conditions}

The Arabidopsis wild type (WT) Col-0 and pepper cold resistance cultivar "P70" were provided by the College of Horticulture, Northwest A\&F University, Yangling China. The transgenic Arabidopsis and pepper plants were grown in a growth chamber at $22{ }^{\circ} \mathrm{C}$ (day for $16 \mathrm{~h}$ ) $/ 18^{\circ} \mathrm{C}$ (night for $8 \mathrm{~h}$ ) and a relative humidity of $70 \%$. The $\mathrm{T} 3$ transgenic Arabidopsis and WT seeds were sterilized in 75\% (v/v) ethanol for $30 \mathrm{~s}$, treated with $5 \%$ $(v / v) \mathrm{NaClO}$ for $10 \mathrm{~min}$, then four washes with water. The seeds were vernalized at $4{ }^{\circ} \mathrm{C}$ for $1 \mathrm{~d}$, then placed on $1 / 2$ MS medium solidified.

\subsection{RNA Extraction and QPCR Analysis}

Plant total RNA was extracted according to manufacturer's protocol (Tian Gen, Xi'an, China), while the qRT-PCR and single cDNA synthesis were proceeded as described by Guo et al. [39]. The iQ5.0 Bio-Rad iCycler thermocycler (BioRad, Hercules, CA, USA) was used for QPCR. The pepper ubiquitin-binding gene CaUBI3 was used as reference in pepper and Arabidopsis Atactin2 was used as reference in Arabidopsis. The relative expression levels were measured through the $2^{-\Delta \Delta C T}$ method [40], and the experiments were conducted with three biological replicates. All the primers used for the qRT-PCR were shown in the supplementary Table S1.

\subsection{VIGS of Pepper CaDHN3}

The VIGS assay was conducted according to our previous study [41]. Briefly, a 300-bp fragment of the $3^{\prime}$-untranslated region of CaDHN3 was inserted into the pTRV2 vector for the construction of recombinant plasmid pTRV2: CaDHN3. Subsequently, through freeze-thaw method the pTRV1, pTRV2 (negative control), pTRV2: CaPDS (positive control) and pTRV2: CaDHN3 was transformed into an Agrobacterium tumefaciens strain (GV3101). All the above-mentioned vectors (pTRV2, pTRV2-CaPDS and pTRV2-CaDHN3) were mixed at a 1:1 ratio with A. tumefaciens carrying pTRV1. Pepper plants cotyledons were infiltrated with inocula of agrobacterium suspensions $(\mathrm{OD} 600=1.0)$ through $1.0 \mathrm{~mL}$ sterilized needleless syringe. The injected plants were grown at $18{ }^{\circ} \mathrm{C}$ in zan entirely dark environment for 2 days, and then transfer into normal growing conditions $\left(22^{\circ} \mathrm{C}\right.$ at day 
for $16 \mathrm{~h} / 18^{\circ} \mathrm{C}$ at night for $8 \mathrm{~h}$ ) with $50 \%$ relative humidity. About 30 days later, the control pepper plants (pTRV2:CaPDS and pTRV2:00) and silenced plants (TRV2:CaDHN3) were analyzed for calculating the silencing efficiency.

\subsection{Transient Expression in Arabidopsis Plants}

To obtain the transgenic Arabidopsis plants, the full-length of CaDHN3 was obtained from pepper cDNA and cloned into the pVBG2307 vector and the recombinant plasmid pVBG2307-CaDHN3 was constructed. Transgenic lines were developed through the floral dipping methods and screened by $1 / 2 \mathrm{MS}$ solid medium accompanied by $50 \mathrm{mg} / \mathrm{L}$ kanamycin, and subsequently, the T3 generations were used for further experiments.

\subsection{Salt and Drought Assays}

For analysis of salt and drought stress tolerance, two transgenic lines and the WT plants were selected. The WT and transgenic lines were grown on MS medium having $\mathrm{NaCl}(0,100 \mathrm{mM}, 150 \mathrm{mM})$ and mannitol $(0,150 \mathrm{mM}, 200 \mathrm{mM})$ respectively, and assessed for seed germination. The root length and the fresh weight of the WT and transgenic lines of the above-mentioned treatments were determined 7th days, while plants growing condition was $16 \mathrm{~h}$ light $/ 8 \mathrm{~h}$ dark cycle at $22^{\circ} \mathrm{C} / 18^{\circ} \mathrm{C}$.

For salt stress, the control (pTRV2:00) and silenced (pTRV2:CaDHN3) pepper plants were treated with $300 \mathrm{mM} \mathrm{NaCl}$ for 3 days. For drought analysis, plants were exposed to drought stress without water for 7 days. Then photographs were taken and the collected samples were stored at $-80^{\circ} \mathrm{C}$ for further use.

To further understand the response of transgenic Arabidopsis to salt and drought stress, about 3-week-old T3 transgenic and WT Arabidopsis lines were treated with salt or drought stresses. For salt treatment, plants were watered with $300 \mathrm{mM} \mathrm{NaCl}$ for 15 days at least 30 plants for each line and watering with salt solution was carried out at 3-day intervals. For drought treatment, plants suffered from drought stress by going without water for 15 days. At least 30 plants for each line were taken and then re-watered for 3 days. Phenotypic changes in Arabidopsis plants were found and photographed during salt and drought treatment periods. Samples of biochemical parameters measurement were measured 10 days after salt and drought treatments. Normal conditions grown plants were used as a positive control.

\subsection{Measurement REL, Chlorophyll Content and MDA Content}

The REL was estimated as described by Dionisio-Sese and Tobita [42]. Total chlorophyll was tested spectrophotometrically. Leaves were collected and incubated in 95\% ethyl alcohol and absorbance was measured at 663 and $646 \mathrm{~nm}$, as described by Lichtenthaler and Wellburn et al. [43]. The MDA contents were measured according to the method of Campos et al. [44] with minor modifications. The crude enzyme used for MDA contents was extracted by $10 \%$ trichloroacetic acid (TCA). Then, $2 \mathrm{~mL}$ of the crude enzyme extract was mixed with $2 \mathrm{~mL}$ of $0.6 \%$ thiobarbituric acid (TBA), boiled for $10 \mathrm{~min}$, quickly cooled, and centrifuged at $12,000 \times g$ for $10 \mathrm{~min}$. Absorbance was analyzed at 600, 532, and $450 \mathrm{~nm}$.

\subsection{Measurement of $\mathrm{H}_{2} \mathrm{O}_{2}$ and $\mathrm{O}_{2}{ }^{\bullet-}$ Contents, Histochemical Detection of ROS and Antioxidant Enzymes Activities}

$\mathrm{H}_{2} \mathrm{O}_{2}$ content was determined according to Chakrabarty et al. [45]. Briefly, the samples were ground with $0.1 \%$ TCA $(w / v)$. After grinding, the homogenate was centrifuged at $12,000 \times g$ for $15 \mathrm{~min}$, the supernatant $0.5 \mathrm{~mL}$ was added to $2 \mathrm{~mL} 1 \mathrm{M} \mathrm{KI}$ and $0.5 \mathrm{~mL} 100 \mathrm{mM}$ potassium sulfate buffer and kept in dark for $1 \mathrm{~h}$. The absorption value at $390 \mathrm{~nm}$ was determined. $\mathrm{O}_{2}{ }^{\bullet-}$ contents were estimated as described by Ke et al. [46]. The SOD and POD activity was determined as previously described by Stewart and Jariteh et al. [47,48]; the CAT activity was assayed using ultraviolet spectrophotometry [49]; the APX activity was assayed as previously described Mittova et al. [50]. 


\subsection{NBT and DAB Staining}

To detect the $\mathrm{H}_{2} \mathrm{O}_{2}$ and superoxide $\left(\mathrm{O}_{2}{ }^{-}-\right)$under salt and drought stresses, 3,3'diaminobenzidine (DAB) and nitro-blue tetrazolium (NBT) staining were carried out. After staining, the leaves were soaked in $75 \%$ ethanol. After bleaching, images were taken immediately as anteriorly described by Able et al. [51]. The quantification of the DAB and NBT strained areas was obtained using the method described by Sekulska-Nalewajko et al. [52].

\subsection{Measurement of Stomatal Aperture}

Stomatal apertures after salt and drought stress were measured as described by Zhang et al. [53]. The 3-week-old T3 transgenic and WT plants were exposed to $300 \mathrm{mM}$ $\mathrm{NaCl}$ and without water for $7 \mathrm{~d}$, respectively. Plants under normal conditions were regarded as a control. The leaves from the same location were chosen and then immediately images were taken. The stomatal apertures were determined by using Image J software (Rawak Software Inc., Stuttgart, Germany). Stomatal aperture values of at least 50 stomata were measured.

\subsection{Sub-Cellular Localization, Yeast Two-Hybrid (Y2H) and Bimolecular Fluorescence Complementation (BiFC) Assay}

Sub-cellular localization was analyzed as described by Zhang et al. [45]. The transcriptional activity of the CaDHN3 protein was analyzed with the yeast two-hybrid assay. For $\mathrm{Y} 2 \mathrm{H}$ assay the cDNA fragments of CaDHN3 were cloned into pGBKT7 vectors. Similarly, cDNA fragments of CaHIRD11 were cloned into pGADT7 vectors. The Co-transformed CaDHN3-BD and CaHIRD11-AD were spotted in SD-Leu-Trp, SD/-Trp/-Leu/-His/-Ade. Yeast growth was performed at $30^{\circ} \mathrm{C}$ for about $3-5$ days to authenticate the interactions between $\mathrm{CaDHN} 3$ and CaHIRD11 proteins.

For BiFC assays, CaDHN3 was linked to the C-terminal of YFP; the dehydrin gene of CaHIRD11 was fused to the N-terminal of YFP. We performed the recombination plasmid transform into GV3101, which was then injected into tobacco leaves. The fluorescence was found after $48 \mathrm{~h}$ by fluorescence microscopy (Olympus Corporation, Tokyo, Japan).

\subsection{Statistical Analysis}

Data were analyzed through a $t$-test. The mean \pm standard deviation (SD) originates from the average of three biological replicates and remarkable differences compare to the control group are marked at ${ }^{*} p<0.05$.

\section{Conclusions}

In conclusion, we isolated a $D H N$ gene $(C a D H N 3)$ from pepper. Ectopic expression of the CaDHN3 in Arabidopsis enhanced resistance to salt and drought stresses through increase in the seedling root length, germination rate and protection of the cell membrane, while silencing of the CaDHN3 decreased the salt and drought stress tolerance by reduced ROS scavenging and through lowered expression of stress- and antioxidant-related genes and antioxidant enzymes activities. According to the BiFC results, we found CaDHN3 interacted with CaHIRD11 in the plasma membrane. Further studies are suggested to focus on the regulatory mechanism of $\mathrm{CaDHN} 3$ in better understanding the molecular mechanisms of abiotic stress tolerance Figure 10. 


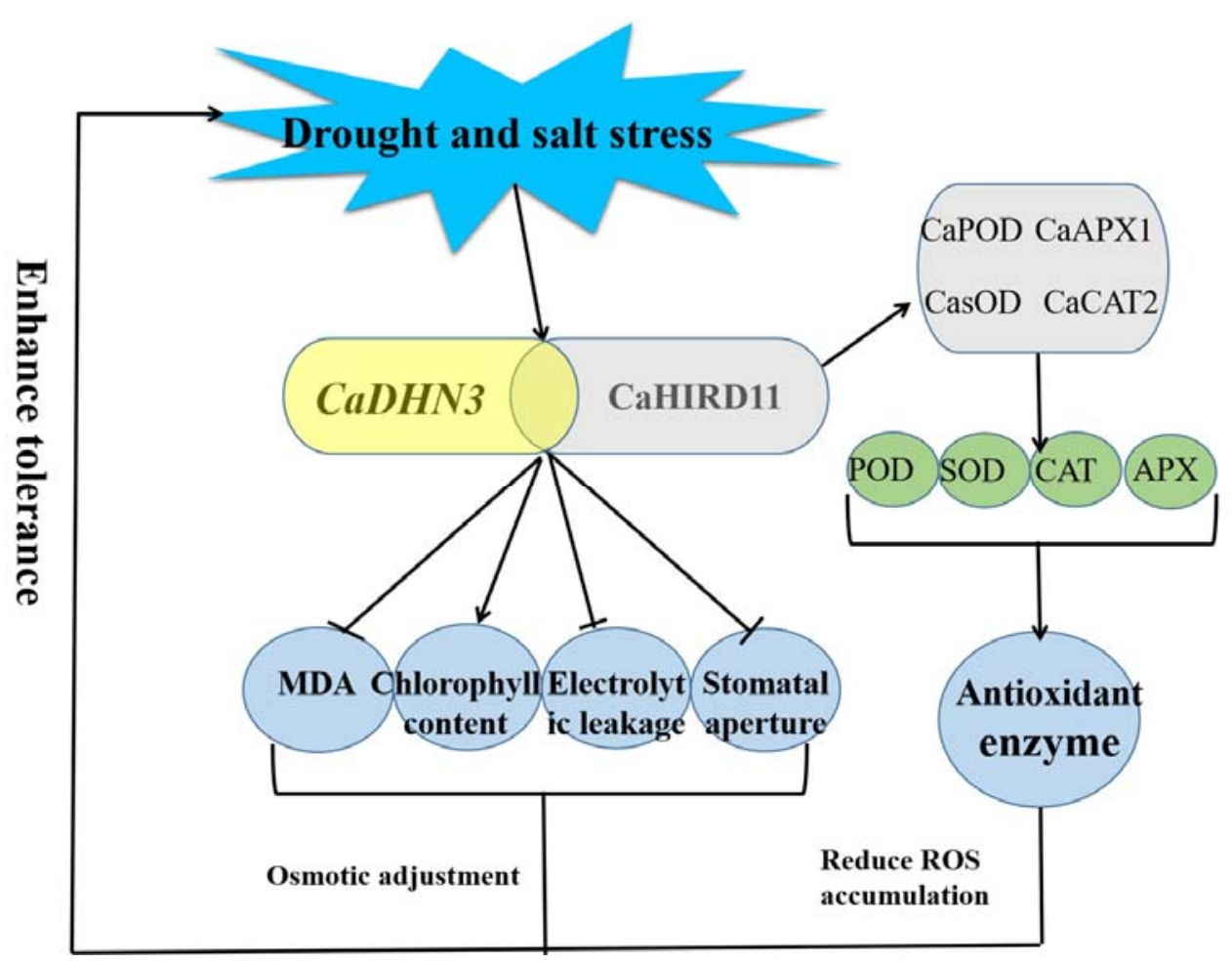

Figure 10. Schematic illustration of the mechanism of salt and drought tolerance regulated by CaDHN3 in pepper.

Supplementary Materials: The following are available online at https:/ /www.mdpi.com/1422-006 7/22/6/3205/s1, Figure S1: The phenotypes and analysis of CaDHN3 expression of silencing pepper plants, Table S1: Primers were used for the qRT-PCR.

Author Contributions: R.-G.C. conceived and designed the experiments; Y.-C.M., X.-X.P., H.F.Z. and H.-F.H. performed the experiments; S.u.H., A.K. analyzed the data; N.C. contributed reagents/materials/analysis tools; H.-F.Z. wrote the paper. All authors have read and agreed to the published version of the manuscript.

Funding: This research was funded by the National Natural Science Foundation of China (\#31672146, \#31201615) and the Natural Science Foundation of Shaanxi Province (2018JM3023).

Institutional Review Board Statement: Not applicable.

Informed Consent Statement: Not applicable.

Data Availability Statement: Not applicable.

Conflicts of Interest: The authors declare no conflict of interest.

\section{References}

1. Mittler, R. Abiotic stress, the field environment and stress combination. Trends Plant Sci. 2006, 11, 15-19. [CrossRef]

2. Bartels, D.; Sunkar, R. Drought and Salt Tolerance in Plants. Crit. Rev. Plant Sci. 2005, 24, 23-58. [CrossRef]

3. Shinozaki, K.; Yamaguchi-Shinozaki, K. Gene networks involved in drought stress response and tolerance. J. Exp. Bot. 2007, 58, 221-227. [CrossRef] [PubMed]

4. Kim, S.; Park, M.; Yeom, S.I. Genome sequence of the hot pepper provides insights into the evolution of pungency in Capsicum spp. Nat. Genet. 2014, 46, 270-278. [CrossRef] [PubMed]

5. Sun, X.; Lin, H.H. Role of plant dehydrins in antioxidation mechanisms. Biologia 2010, 65, 755-759. [CrossRef]

6. Graether, S.P.; Boddington, K.F. Disorder and function: A review of the dehydrin protein family. Front. Plant Sci. $2014,5,576$. [CrossRef] [PubMed]

7. Enebe, M.C.; Babalola, O.O. The influence of plant growth-promoting rhizobacteria in plant tolerance to abiotic stress: A survival strategy. Appl. Microbiol. Biotechnol. 2018, 102, 7821-7835. [CrossRef] [PubMed]

8. Allagulova, C.R.; Gimalov, F.R.; Shakirova, F.M.; Vakhitov, V.A. The Plant Dehydrins: Structure and Putative Functions. Biochemistry 2003, 68, 945-951. [CrossRef] 
9. Koag, M.C.; Wilkens, S.; Fenton, R.D.; Resnik, J.; Vo, E.; Close, T.J. The K-Segment of Maize DHN1 Mediates Binding to Anionic Phospholipid Vesicles and Concomitant Structural Changes. Plant Physiol. 2009, 150, 1503-1514. [CrossRef]

10. Hughes, S.L.; Schart, V.; Malcolmson, J.; Hogarth, K.A.; Martynowicz, D.M.; Tralman-Baker, E. The importance of size and disorder in the cryoprotective effects of dehydrins. Plant Physiol. 2013, 163, 1376-1386. [CrossRef] [PubMed]

11. Close, T.J. Dehydrins: Emergence of a biochemical role of a family of plant dehydration proteins. Plant Physiol. 1996, 97, 795-803. [CrossRef]

12. Close, T.J. Dehydrins: A commonality in the response of plants to dehydration and low temperature. Plant Physiol. 1997, 100, 291-296. [CrossRef]

13. Nguyen, P.N.; Tossounian, M.-A.; Kovacs, D.S.; Thu, T.T.; Stijlemans, B.; Vertommen, D.; Pauwels, J.; Gevaert, K.; Angenon, G.; Messens, J.; et al. Dehydrin ERD14 activates glutathione transferase Phi9 in Arabidopsis thaliana under osmotic stress. Biochim. Biophys. Acta Gen. Subj. 2020, 1864, 129506. [CrossRef]

14. Lv, A.; Fan, N.; Xie, J.; Yuan, S.; An, Y.; Zhou, P. Expression of CdDHN4, a Novel YSK2-Type Dehydrin Gene from Bermudagrass, Responses to Drought Stress through the ABA-Dependent Signal Pathway. Front. Plant Sci. 2017, 8, 748. [CrossRef]

15. Guo, X.; Zhang, L.; Wang, X.; Zhang, M.; Xi, Y.; Wang, A.; Zhu, J. Overexpression of Saussurea involucrata dehydrin gene SiDHN promotes cold and drought tolerance in transgenic tomato plants. PLoS ONE 2019, 14, e0225090. [CrossRef] [PubMed]

16. Aduse Poku, S.; Nkachukwu Chukwurah, P.; Aung, H.H.; Nakamura, I. Over-Expression of a Melon Y3SK2-Type LEA Gene Confers Drought and Salt Tolerance in Transgenic Tobacco Plants. Plants 2020, 9, 1749. [CrossRef]

17. Kumar, M.; Lee, S.C.; Kim, J.Y.; Kim, S.J.; Aye, S.S.; Kim, S.R. Over-expression of dehydrin gene, OsDhn1, improves drought and salt stress tolerance through scavenging of reactive oxygen species in rice (Oryza sativa L.). J. Plant Biol. 2014, 57, 383-393. [CrossRef]

18. Li, X.; Liu, Q.; Feng, H.; Deng, J.; Zhang, R.; Wen, J.; Dong, J.; Wang, T. Dehydrin MtCAS31 promotes autophagic degradation under drought stress. Autophagy 2019, 16, 862-877. [CrossRef]

19. Cao, Y.; Xiang, X.; Geng, M.; You, Q.; Huang, X. Effect of HbDHN1 and HbDHN2 Genes on Abiotic Stress Responses in Arabidopsis. Front. Plant Sci. 2017, 8, 470. [CrossRef]

20. Halder, T.; Upadhyaya, G.; Ray, S. YSK2 Type Dehydrin (SbDhn1) from Sorghum bicolor Showed Improved Protection under High Temperature and Osmotic Stress Condition. Front. Plant Sci. 2017, 8, 918. [CrossRef] [PubMed]

21. Jing, H.; Li, C.; Ma, F.; Ma, J.H.; Abid, K.; Wang, X.; Zhao, L.Y.; Gong, Z.H.; Chen, R.G. Genome-Wide Identification, Expression Diversification of Dehydrin Gene Family and Characterization of CaDHN3 in Pepper (Capsicum annuum L.). PLoS ONE 2016, 8, e0161073.

22. Alexandersson, E.; Saalbach, G.; Larsson, C.; Kjellbom, P. Arabidopsis Plasma Membrane Proteomics Identifies Components of Transport, Signal Transduction and Membrane Trafficking. Plant Cell Physiol. 2004, 45, 1543-1556. [CrossRef] [PubMed]

23. Borovskii, G.B.; Stupnikova, I.V.; Antipina, A.I.; Vladimirova, S.V.; Voinikov, V.K. Accumulation of dehydrin-like proteins in the mitochondria of cereals in response to cold, freezing, drought and ABA treatment. BMC Plant Biol. 2002, 2, 5. [CrossRef]

24. Salleh, F.M.; Evans, K.; Goodall, B.; Machin, H.; Mowla, S.B.; Mur, L.A.J.; Runions, J.; Theodoulou, F.L.; Foyer, C.H.; Rogers, H.J. A novel function for a redox-related LEA protein (SAG21/AtLEA5) in root development and biotic stress responses. Plant Cell Environ. 2012, 35, 418-429. [CrossRef] [PubMed]

25. Mueller, J.K.; Heckathorn, S.A.; Fernando, D. Identification of a Chloroplast Dehydrin in Leaves of Mature Plants. Int. J. Plant Sci. 2003, 164, 535-542. [CrossRef]

26. Xing, X.; Liu, Y.; Kong, X.; Liu, Y.; Li, D. Overexpression of a maize dehydrin gene, ZmDHN2b, in tobacco enhances tolerance to low temperature. Plant Growth Regul. 2011, 65, 109-118. [CrossRef]

27. Lee, S.C.; Kim, S.H.; Kim, S.R. Drought inducible OsDhn1 promoter is activated by OsDREB1A and OsDREB1D. J. Plant Biol. 2013, 56, 115-121. [CrossRef]

28. Zhu, W.N.; Zhang, D.P.; Lu, X.X.; Zhang, L.S.; Yu, Z.Y.; Lv, H. Characterization of an SKn-type dehydrin promoter from wheat and its responsiveness to various abiotic and biotic stresses. Plant Mol. Biol. Rep. 2014, 32, 664-678. [CrossRef]

29. Qin, Y.X.; Qin, F. Dehydrins from wheat $x$ Thinopyrum ponticum amphiploid increase salinity and drought tolerance under their own inducible promoters without growth retardation. Plant Physiol. Biochem. 2016, 99, 142-149. [CrossRef]

30. Wisniewski, M.; Webb, R.; Balsamo, R.; Close, T.J.; Yu, X.M.; Griffith, M. Purification, immunolocalization, cryoprotective, and antifreeze activity of PCA60: A dehydrin from peach (Prunus persica). Physiol. Plant. 1999, 105, 600-608. [CrossRef]

31. Lin, C.H.; Peng, P.H.; Ko, C.Y.; Markhart, A.H.; Lin, T.Y. Characterization of a Novel Y2K-type Dehydrin VrDhn1 from Vigna radiata. Plant Cell Physiol. 2012, 53, 930-942. [CrossRef]

32. Danyluk, J.; Perron, A.; Houde, M.; Limin, A.; Fowler, B.; Benhamou, N. Accumulation of an acidic dehydrin in the vicinity of the plasma membrane during cold acclimation of wheat. Plant Cell 1998, 10, 623-638. [CrossRef] [PubMed]

33. Hara, M.; Terashima, S.; Fukaya, T.; Kuboi, T. Enhancement of cold tolerance and inhibition of lipid peroxidation by citrus dehydrin in transgenic tobacco. Planta 2003, 217, 290-298. [CrossRef] [PubMed]

34. Mafakheri, A.; Siosemardeh, A.; Bahramnejad, B.; Struik, P.C.; Sohrabi, Y. Effect of drought stress on yield, proline and chlorophyll contents in three chick pea cultivars. Aust. J. Crop Sci. 2010, 4, 580-585.

35. Moore, K.; Roberts, L.J. Measurement of Lipid Peroxidation. Free Radic. Res. 1998, 28, 659-671. [CrossRef] [PubMed]

36. Xie, C.; Zhang, R.; Qu, Y.; Miao, Z.; Zhang, Y.; Shen, X.; Wang, T.; Dong, J. Overexpression of MtCAS31 enhances drought tolerance in transgenic Arabidopsis by reducing stomatal density. New Phytol. 2012, 195, 124-135. [CrossRef] 
37. Liu, Y.; Wang, L.; Zhang, T.; Yang, X.; Li, D. Functional characterization of KS-type dehydrin ZmDHN13 and its related conserved domains under oxidative stress. Sci. Rep. 2017, 7, 7361. [CrossRef]

38. Yokoyama, T.; Ohkubo, T.; Kamiya, K.; Hara, M. Cryoprotective activity of Arabidopsis KS-type dehydrin depends on the hydrophobic amino acids of two active segments. Arch. Biochem. Biophys. 2020,691, 108510. [CrossRef]

39. Guo, W.L.; Chen, R.G.; Gong, Z.H.; Yin, Y.X.; Li, D.W. Suppression Subtractive Hybridization Analysis of Genes Regulated by Application of Exogenous Abscisic Acid in Pepper Plant (Capsicum annuum L.) Leaves under Chilling Stress. PLoS ONE 2013, 8, e66667. [CrossRef] [PubMed]

40. Livak, K.J.; Schmittgen, T.D. Analysis of relative gene expression data using real-time quantitative PCR and the $2^{-\Delta \Delta C T}$ method. Methods 2001, 25, 402-408. [CrossRef]

41. Khan, A.; Li, R.J.; Sun, J.T.; Ma, F.; Zhang, H.X.; Jin, J.H.; Ali, M.; Haq, S.U.; Wang, J.E.; Gong, Z.-H. Genome-wide analysis of dirigent gene family in pepper (Capsicum annuum L.) and characterization of CaDIR7 in biotic and abiotic stresses. Sci. Rep. 2018, 8, 5500. [CrossRef] [PubMed]

42. Dionisio-Sese, M.L.; Tobita, S. Antioxidant responses of rice seedlings to salinity stress. Plant Sci. 1998, 135, 1-9. [CrossRef]

43. Lichtenthaler, H.K.; Wellburn, A.R. Determinations of total carotenoids and chlorophylls a and b of leaf extracts in different solvents. Biochem. Soc. Trans. 1983, 11, 591-592. [CrossRef]

44. Campos, P.S.; Quartin, V.N.; Ramalho, J.C.; Nunes, M.A. Electrolyte leakage and lipid degradation account for cold sensitivity in leaves ofCoffea sp. plants. J. Plant Physiol. 2003, 160, 283-292. [CrossRef]

45. Chakrabarty, D.; Datta, S.K. Micro propagation of gerbera: Lipid peroxidation and antioxidant enzyme activities during acclimatization process. Plant Physiol. 2008, 30, 325-331.

46. Ke, D.; Sun, G.; Wang, Z. Effects of superoxide radicals on ACC synthase activity in chilling-stressed etiolated mungbean seedlings. Plant Growth Regul. 2007, 51, 83-91. [CrossRef]

47. Stengel, F.; Baldwin, A.J.; Painter, A.J.; Jaya, N.; Basha, E.; Kay, L.E.; Vierling, E.; Robinson, C.V.; Benesch, J.L.P. Quaternary dynamics and plasticity underlie small heat shock protein chaperone function. Proc. Natl. Acad. Sci. USA 2010, 107, $2007-2012$. [CrossRef]

48. Jariteh, M.; Ebrahimzadeh, H.; Niknam, V. Developmental changes of protein, proline and some antioxidant enzymes activities in somatic and zygotic embryosof Persian walnut (Juglans regia L.). Plant Cell Tissue Organ Cult. 2015, 122, 101-115. [CrossRef]

49. Aebi, H. Catalase in vitro. Meth. Enzymol. 1984, 105, 121-126.

50. Mittova, V.; Volokita, M.; Guy, M.; Tal, M. Activities of SOD and the ascorbate-glutathione cycle enzymes in subcellular compartments in leaves and roots of the cultivated tomato and its wild salt-tolerant relative Lycopersicon pennellii. Physiol. Plant. 2000, 110, 42-51. [CrossRef]

51. Able, A.J. Role of reactive oxygen species in the response of barley to necrotrophic pathogens. Protoplasma 2003, 221, 137-143. [CrossRef] [PubMed]

52. Sekulska-Nalewajko, J.; Gocławski, J.; Chojak-Kozniewska, J. Automated image analysis for quantification of reactive oxygen species in plant leaves. Methods 2016, 109, 114-122. [CrossRef] [PubMed]

53. Zhang, H.; Ma, F.; Wang, X.; Liu, S.; Saeed, U.H.; Hou, X.; Zhang, Y.; Luo, D.; Meng, Y.; Zhang, W.; et al. Molecular and Functional Characterization of CaNAC035, an NAC Transcription Factor From Pepper (Capsicum annuum L.). Front. Plant Sci. 2020, 11, 14. [CrossRef] [PubMed] 